\title{
Benefit and Evaluation of Interactive 3D Process Data Visualization for the Presentation of Complex Problems
}

\author{
Dorothea Pantförder ${ }^{1}$, Birgit Vogel-Heuser ${ }^{1}$, and Karin Schweizer ${ }^{2}$ \\ ${ }^{1}$ Chair of Embedded Systems, Faculty of Electrical Engineering/Computer Science, \\ University of Kassel, Wilhelmshöher Allee 73, 34121 Kassel, Germany \\ \{pantfoerder, vogel-heuser\} @uni-kassel.de \\ ${ }^{2}$ Faculty of Social Sciences, University of Mannheim, \\ Schloss, Ehrenhof Ost, 68131 Mannheim, Germany \\ kschweizat-online.de
}

\begin{abstract}
The increasing complexity of industrial plants and more intelligent equipment technology lead to a growing amount of process data. The approach of interactive $3 \mathrm{D}$ process data visualization and associated training concepts for processes without a process model can assists operators to analyze complex processes. This paper describes a set of experiments that analyzed the benefits of a 3D data presentation as part of an HMI in combination with different types of operator training in process control.
\end{abstract}

Keywords: 3D visualization, operator training.

\section{Motivation}

One of the main questions in industry in the recent years is how to support the operator in monitoring and to controlling processes that are getting more and more complex. If the information is not preprocessed, more intelligent equipment technology may lead to an overload of information. Centralized control rooms and the reduction of operating personal increases the operator's work load. Finally, this can result in a rising error rate. Common 2D concepts in HMI and training may not support the operator sufficiently. The 3D process data visualization approach can support the operator in handling large amounts of data. By coding the data, e.g. through color or surface design, the information content of the visualization can be increased. By means of coloring and surface design it is possible to show more data in one view and to visualize potential coherences. Previous works showed the reduction of the number of visualization screens when using 3D [1].

3D data visualization can also be applied in training to support the operator in developing process knowledge. A 3D data player, a so called 3D slider, can be used to teach process dynamic to the operator on basis of real process data for processes without a process model. The operator is able to learn the process behavior in critical situations during the training phase and to identify similar situations in the operating phase.

Up to date, there are no detailed evaluation for 3D process data visualization in process control and operator training. This paper describes an empirical evaluation 
that was conducted within the project PAST- $\mathrm{P}^{1}$ to show the benefits of $3 \mathrm{D}$ visualization in process control.

\section{State of the Art}

In the following we describe the state of the art in 3D process data visualization and operator training.

\subsection{D in Process Data Visualization and the Psychological Background}

Current HMI systems in process control use 2D representations like line diagrams and tables to visualize process data. These visualization elements are available in libraries and can be integrated into the process screen and be connected with process variables in a simple way. HMI systems also offer 3D representations of tanks and piping but this kind of representation has no additional benefit. Beuthel [2] and Hoppe [3] proved the advantages of $3 \mathrm{D}$ process data visualizations for special applications. Both studies measured the reaction time and the processing time to handle problem situations in $2 \mathrm{D}$ as compared with $3 \mathrm{D}$ visualizations. The results showed an advantage for 3D representation.

3D data visualization, like it is common in e.g. Matlab or Excel, is rarely used in process control. The time consuming development could be a reason for this. Each $3 \mathrm{D}$ scene has to be programmed step by step. For this reason, we developed a prototype library with $3 \mathrm{D}$ objects, so called $3 \mathrm{D}$ pattern [4]. These 3D patterns use standard interfaces like OPC or ActiveX and can be integrated in most state-of-the art HMI systems as easy as $2 \mathrm{D}$ visualization objects. The library was developed based on real application examples from industry and has been evaluated by experts.

In a pilot test the authors evaluated $2 \mathrm{D}$ and $3 \mathrm{D}$ visualization comparatively to reach a more reliable answer regarding the advance of 3D [5]. The results showed significant faster reaction time in the $3 \mathrm{D}$ environment if the problem situation was complex. This result corresponds with Wickens' "proximity-Compatibility"- principle [6]. This principle states that tasks that require the integration of information, benefit from a perceptual proximity of the display or visualization. The integration of information is necessary in particular for the detection of complex problems. These problems require the combination of three or more process values for the analysis of the system status. However, in the pilot test only one out of five problem situations disclose this benefit. One reason for the missing significance for the other problems could be the lack of complexity. The problem situations had to be simplified because subjects, which have no prior knowledge about the process, had to be trained adequately. Compared to real control rooms in industry, the experimental environment also had to be simplified. In the pretest the subjects had to observe only one diagram and could completely concentrate on this task. The task of an operator in a real control room is more complex as he has to observe a few monitors with different kinds of diagrams and tables. Furthermore, he has to attend to additional tasks that will distract him from his main task and by that reduces his attention to the process monitoring task.

${ }^{1}$ The project is funded by DFG (German Research Foundation under VO 937/7-1 and SCHW $736 / 3-1$ from $5 / 2007$ to $4 / 2009$. 
As 3D visualization is displayed on $2 \mathrm{D}$ screens the topic of interaction also has to be taken into account. Only by means of interactions like translation or rotation of elements can the 3D effect be perceived completely. Schönhage's [7] approach is based on the DIVA Java 3D-collection and implements five different kinds of interactions, so called behaviors. Amongst others, Schönhage provides interaction that enables to display additional information by mouse clicks / keystrokes or actions that move or rotate the $3 \mathrm{D}$ elements.

According to Witmer and Singer [8] interaction with a 3D scene also increases the sense of presence. Presence is defined as the sense of being present in a virtual environment, i.e. effects that influence the cognition of a state, the bonding to an event or the immersion into the virtual environment also influence the feeling of presence. Witmer and Singer specify four main factors. Two of them can be recognized as relevant for the task of monitoring process data:

- Control factors: degree of control, immediacy of control, anticipation of events, and mode of control and modifiability of physical environment.

- Sensory factors: sensory modality, environmental richness, multimodal presentation, consistency of multimodal information, degree of movement perception, active search.

The results of the pretest as well as the studies of Wickens on the complexity and Witmer and Singer on presence are the basis for our further experiments. These experiments have to be more realistic and therefore will be more complex.

\subsection{Operator Training -Learning of Process Behavior}

Increasing complexity of the experiment also takes up new training strategies. The pretest training is no longer sufficient to train the subjects for the more complex situations. In chemistry or pharmaceutical industry an operator is often trained by means of an operator training system (OTS) where a model based description of the process is available [9]. To learn process knowledge, the process dynamics need to be modeled in the OTS. The OTS systems use the original HMI interface of the process, which have to be learned, to display the system status and allow the operator to interact with the process. A simulation of the process calculates the new system status based on the actual state and the operators' intervention.

However, for a lot of processes it is not possible to identify the process model with an acceptable effort. In this cases operator training is often realized as training on the job. Novice operators are either trained by the start-up personnel or by an experienced operator based on their own mental model of the process. The 3D slider that was developed by the authors could be an alternative approach to teach the operator. Klein [11] showed that engineers often use knowledge from similar and familiar situations to solve a new problem instead of making time-consuming analysis. The 3D slider uses real scenarios and real process data from similar plants for a "scenario-based training" [12]. With the functionality of the slider it is possible to analyze historical data in an adjustable temporal scaling. In doing so the operator learns the process dynamic in familiar situations and can build up a mental model of the process. The training with the $3 \mathrm{D}$ slider requires real data of the process of a comparable plant. Some OTS use the approach by means of a slider in combination with simulated data but not 
with a $3 \mathrm{D}$ visualization. In the experiment we describe in the following three different types of training were evaluated in combination with 2D and 3D visualization.

\section{Hypotheses}

The main task of an operator in process control is the analysis of the actual process status. He has to differentiate between error and error free conditions and to identify the appropriate solution. Two different cases can be defined:

- hit: right identification of error conditions.

- miss: no identification or false identification of an error condition.

The following hypotheses were deduced:

(H1) $3 D$ visualization leads to better error detection in complex problem situations than $2 D$ visualization.

According to Wickens a 3D representation is beneficial for the detection of errors on complex problems, i.e. if it is necessary to combine at least three process values for the identification of the system state. Hence, for the error detection of complex problems, subjects that use 3D visualization will have more hits as compared to subjects that use $2 \mathrm{D}$ visualization.

(H2) 3D visualization with interaction leads to better error detection in complex problem situations compared with $3 D$ visualization without interaction.

Interaction is a basic control factor that increases the presence. A $3 \mathrm{D}$ visualization with interaction leads to a higher control over the visualization environment and should facilitate the operators' task of error detection. Hence, subjects that use 3D with interaction will have more hits than subjects that use $3 \mathrm{D}$ without interaction.

\section{Experiment}

By analyzing the tasks in the control room of a continuous hydraulic press, the subjects' tasks in the experiment as well as the implementation of the HMI were designed.

\subsection{Application Example}

For the empirical evaluation of the described hypotheses an appropriate application example that is appropriate for $3 \mathrm{D}$ has to be identified. Due to the experiences of the authors the case study of the continuous hydraulic press (Fig. 1) was selected [13]. The hydraulic press is used to produce different kinds of fiber boards. A glued material mat runs into the continuous press where it is pressed between two moving steel belts. The heat, that is necessary for the technological process, is transferred by roller rods from the heating plate to the steel belt. Hydraulic cylinders generate the pressure to press the material mat to the set values of distance. The cylinders are located equally spaced along the whole length and width of the press. The thickness of the mat is measured by means of distance transducers at the outer edges of the steel belts and is controlled by increasing or reducing the pressure. Important data, like 

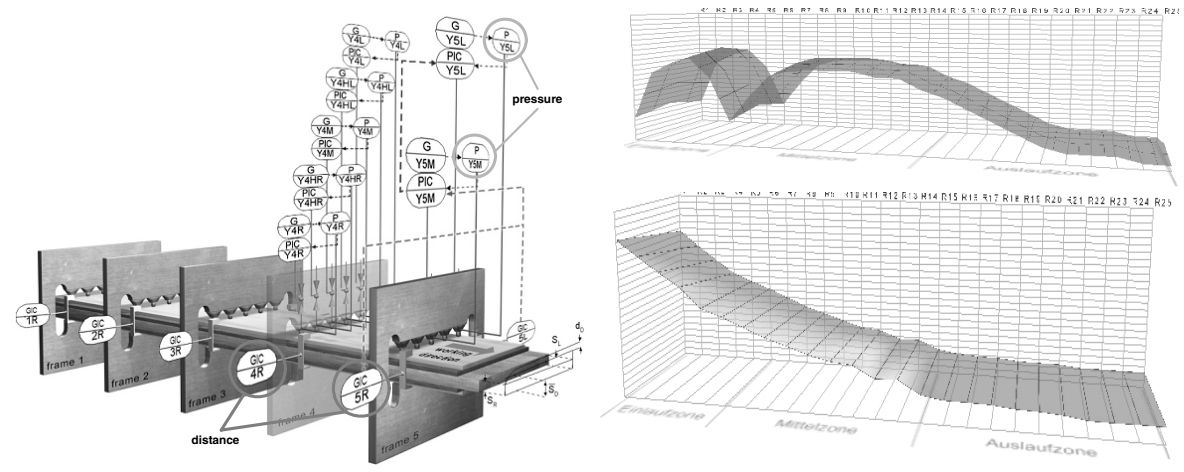

Fig. 1. P\&ID of the continuous hydraulic press and associated 3D visualization of pressure and distance

temperature of the heating plate, thickness and pressure are continuously measured and displayed for the operator in the control room. Based on the actual process date and his knowledge about the process the operator decides how to take corrective actions on the process.

In the described application example multiple dependencies exist between different process factors, e.g. pressure and distance. The material has to be pressed to the set distance without exceeding the maximum pressure. For decision-making the operator has to observe both process values and set into correlation across and along the press. For the experiment five problem situations from the real application example were chosen: two simple and three complex ones.

\subsection{Design of the Experiment}

The subjects' task in the experiment was to monitor the press. The experiment was divided into three phases: audio-visual training, exploration phase and operation phase.

In the audio-visual phase the subjects were shown a video that explained the application process. The relevant process factors and their interrelations were explained. The critical process situations and their symptoms were described. Furthermore, the video gave an introduction to the process visualization and the options of taking corrective actions.

Next, the subjects enter into the exploration phase. Firstly, the subjects reconstruct the information from the video and comprehend the process behavior and the scenario based on simulated process data. In the second step, the subjects verify the obtained knowledge in the testing phase where they had to operate the process and attain feedback concerning the correctness of their interventions in the experimental environment.

In the operation phase, the subjects had to control the process. They had to decide whether they were confronted with normal or with critical process conditions. In case of a critical situation they had to take an appropriate corrective action. During the observation and control of the process they had to execute two additional tasks, i.e. hand writing product data on a protocol data sheet and communicating with colleagues in a chat instead of a walkie-talkie in a real plant (referring to Wicken's theory a second 
task was introduced, indicates the work load performance in secondary task). The subjects had to undergo the same selection of situations twice in random order. All actions of the subjects, e.g. error response time, were automatically recorded by the system. Additionally, the heart rate was measured during the experiment to evaluate physiological factors like stress.

To date, the experiment was conducted with 70 subjects (students of different fields of study). All subjects had no previous knowledge about the process. To allow the comparison between $2 \mathrm{D}$ and $3 \mathrm{D}$ visualizations as well as the comparison of the different variations of training, the subjects were divided randomly into five groups, each group with 14 subjects (Table 1). The training conditions were derived to examine three different modes of presence, the lowest mode: a classical training (freeze image in $2 \mathrm{D}$ or $3 \mathrm{D}$ ) with figures showing critical situations and giving verbal instructions. The slider mode (2D or 3D) displays the development of a critical situation and should by that give more information to detect the critical situation. Training with slider and interaction (only 3D) should generate the highest presence because the interaction gives subjects the opportunity to feel more involved in the process. The training with slider and interaction was evaluated only in $3 \mathrm{D}$ because $2 \mathrm{D}$ does not allow comparable interaction features.

Table 1. Experimental Design (training options and dimension)

\begin{tabular}{lllll}
\hline Dimension of HMI & $\begin{array}{l}\text { Training } \\
\text { image }\end{array}$ & $\begin{array}{l}\text { with freeze } \\
\text { slider }\end{array}$ & $\begin{array}{l}\text { Training } \\
\text { sith }\end{array}$ & $\begin{array}{l}\text { Training with slider } \\
\text { and interaction }\end{array}$ \\
\hline 2D & group 1 & group 2 & -- \\
3D & group 3 & group 4 & group 5 \\
\hline
\end{tabular}

Additionally, different questionnaires were included to examine attentiveness, selfassessment and feedback on presence etc. After the operation phase an interview was conducted to analyze the individual mental models.

\subsection{Experimental Environment}

For the evaluation an experimental environment, which is as realistic as possible, was developed. The main challenge was to find an optimum between the approximation to reality and an environment that does not overstrain novice subjects.

The environment is based on a PC with two monitors displaying four different diagrams. Depending on the provided scenario the subjects had to combine the information displayed in these diagrams to identify the problem and to deduce an appropriate input reaction. On an adjoining tablet PC they had to execute additional tasks, hand writing production protocol. Both PCs were connected and all relevant actions of the subjects on both systems were recorded in one database according to their timeline (more details to the environment are given in [14]).

\section{Results of the Experiment}

The following results refer to the evaluation of 70 subjects. Due to technical problems the results of one subject in group 5 could only be recorded in the first trail; however 
these results were included in the analysis. Table 2 gives an overview of the quantity of subjects in the five groups.

Table 2. Assignment of subjects to training variations

\begin{tabular}{llllll}
\hline group & 1 & 2 & 3 & 4 & 5 \\
\hline subjects & 14 & 14 & 14 & 14 & 13,5 \\
\hline
\end{tabular}

(H1) $3 D$ visualization leads to better error detection in complex problem situations than $2 D$ visualization.

For the evaluation of hypothesis 1 , group 1 and group 2 were joined and represent an overall 2D group. In the same way, group 3 and group 4 were joined and represent the 3D group. Group 5 was not taken into consideration for this analysis. Table 3 shows the total number of errors depending on the experimental group (2D and 3D). The analysis of the results show no significant differences (Fisher test $p=0.5$ ) that can be ascribed to the dimension of the visualization.

Table 3. Hits and misses of complex problems depending on the dimension of the HMI

\begin{tabular}{llll}
\hline group & misses & hits & hits [\%] \\
\hline joined 2D & 159 & 121 & 43.21 \\
joined 3D & 158 & 122 & 43.57 \\
\hline
\end{tabular}

As the analysis shows no significant results, instead of joined group, each group will be analyzed individually. In the analysis, two aspects can be observed (Table 4). The group "2D freeze image" shows better results than the group "2D slider". In contrast the group "3D freeze image" obtains poorer results than the "3D slider" group.

Table 4. Frequency distribution of hits and misses of complex problems in different dimensions

\begin{tabular}{llll}
\hline group & misses & Hits & hits [\%] \\
\hline 2D freeze image & 72 & 68 & 48.57 \\
2D slider & 87 & 53 & 37.86 \\
3D freeze image & 86 & 54 & 38.57 \\
3D slider & 72 & 68 & 48.57 \\
\hline
\end{tabular}

The evaluation shows that hypothesis 1 is false.

(H2) 3D visualization with interaction leads to better error detection in complex problem situations than $3 D$ visualization without interaction.

For the evaluation of hypothesis 2 the five groups were compared. The analysis of table 5 shows that the group "3D slider with interaction" achieves the best result. Actually, this is the only group that obtains more hits than misses. 
Table 5. Hits and misses of complex problems depending on different groups

\begin{tabular}{llll}
\hline group & misses & hits & hits [\%] \\
\hline 2D freeze image & 72 & 68 & 48.57 \\
2D slider & 87 & 53 & 37.86 \\
3D freeze image & 86 & 54 & 38.57 \\
3D slider & 72 & 68 & 48.57 \\
3D slider with interaction & 62 & 73 & 52.14 \\
\hline
\end{tabular}

As the results show significance (chi-square $=11.08, \mathrm{df}=4, \mathrm{p}<0.05$ ), hypothesis 2 can be proven.

\section{Discussion of the Results}

The analysis of the error rates on complex problems showed that a $3 \mathrm{D}$ visualization achieves the best results if the subject is given the opportunity to interact with the 3D scene. Group 5 was significantly better in error detection. A representation of data in 3D without interaction showed no advantage in the experiment. Contrary to the expectation, in several cases subjects of group 1 showed even better results than the subjects of group 3 or the same results like group 4.

The following reasons can be given for these results:

- The representation of data in a 2D diagram is always still the most well-known and widely-used type of data visualization. Therefore the handling of data representation is intuitive because of user's experience. A $2 \mathrm{D}$ visualization in combination with the slider in the exploration phase does not seem to be intuitive for the subjects. In the operating phase the groups 1 and 2 did not differ.

- The groups 3 and 4 also differ only in the exploration phase and not in the operating phase. Nevertheless in 3D the slider group was better in error recognition. The use of the 3D sliders in the exploration phase seems to be more intuitive for the user. This confirms Wickens' theory.

- Group 3 and 4 had a quasi 3D representation, meaning that it is only a predefined 2D projection of a 3D object. Only the interaction generates the real 3D effect that increases the subject's feeling of having control over the visualization, which refers to the presence phenomenon.

The analysis of hits and misses generally shows a high error rate. On top of that group 5 (3D slider with interaction), which was significantly better than the other groups, shows a number of hits that is barely more than 50 percent. This result leads to the assumption that the training phase has to be improved. The present design of the experiment permits no conclusion whether the subjects understood the process which they had to observe and to control, before they enter into the exploration and the operating phase. 


\section{Summary and Future Prospect}

In this paper an experiment that evaluates the influence of different types of visualization (2D, 3D/ interaction) and training on error detection in process control was described. It was shown that training with the classical $2 \mathrm{D}$ freeze image seems to be very intuitive to the user and leads to good results in the operation phase. Referring to $3 \mathrm{D}$ visualization it was shown that interaction is an important factor. Only the combination of 3D with interaction leads to significantly better results in error detection.

Based on the presented results, it seems to be necessary to evaluate the influence of different kinds of interaction with 3D scenes. Besides the already analyzed behavior, namely "rotation", other types of interaction like "translation", "zoom" or "brushing" (context-sensitive display of additional data) have to be evaluated (with prerequisite if they are useful for the operator's task in this application).

For the development of further experiments, the process knowledge of the subjects has to be tested before they enter into the exploration and the operation phase. It must be ensured that the subjects have understood the process and the process intervention. If the subject achieves a certain level of process knowledge the experiment can be continued. Otherwise, the subject has to be trained and be tested again. Finally, if the subject does not improve, the experiment has to be aborted. This adaption of the experimental design should considerably decrease the error rate.

For a more realistic experimental design, it is necessary to examine more complex technical scenarios because the current results show positive correlation from complexity of the scenario to the benefit of 3D presentation. A more realistic scenario, i.e. a more complex scenario, will highlight the benefit of 3D with interaction as compared to the other training conditions in regards to the reaction time and identification of errors. Additionally a subsequent experiment focusing on elderly operators will be conducted. We expect that elderly people will profit from an adaptable interface, e.g. adaptable interaction etc.

The gained results will lead to the implementation of new functionalities in operator training systems and an improved operator training as well as a guideline for application engineers, who develop these systems.

\section{References}

1. Vogel-Heuser, B., Zeipelt, R.: Nutzen der 3D-Prozessdatenvisualisierung in der industriellen Prozessführung. atp - Automatisierungstechnische Praxis 45(3), 45-50 (2003)

2. Beuthel, C.: Dreidimensionale Prozessvisualisierung zum Führen technischer Anlagen am Beispiel eines Kohlekraftwerkes. PhD Thesis University of Clausthal-Zellerfeld. Papierflieger Verlag, Clausthal-Zellerfeld (1997)

3. Hoppe, S.M., Essenberg, G.R., Wiegmann, D.A., Overbye, T.J.: Three-dimensional displays as an effective visualization technique for power systems monitoring and control. Technical Report. University of Illinois, US (2004)

4. Friedrich, D., Moritz, S., Pantförder, D., Vogel-Heuser, B.: 3D-Patterns for the Data Analysis and Operation of Process Plant. In: International Association of Science and Technology for Development- Human Computer Interaction (IASTED-HCI), Phoenix (2005) 
5. Vogel-Heuser, B., Schweizer, K., van Burgeler, A., Fuchs, Y., Pantförder, D.: Auswirkungen einer dreidimensionalen Prozessdatenvisualisierung auf die Fehlererkennung. Zeitschrift für Arbeitwissenschaft 61(1), 23-34 (2007)

6. Wickens, C.D., Andre, A.D.: Proximity Compatibility and Information Display: Effects of Color, Space, and Objectness on Information Integration. Human Factors 32(1), 61-77 (1990)

7. Schönhage, B., van Ballegooij, A., Eliëns, A.: 3D Gadgets for Business Process Visualization -a case study. In: Proceedings of the 5th symposium on Virtual Reality modelling language (web3D-VRML), Monterey CA, pp. 131-138 (2000)

8. Witmer, B.G., Singer, M.J.: Measuring Presence in Virtual Environments: A Presence Questionnaire. Presence 7(3), 225-240 (1998)

9. Kroll, A.: Status and perspectives of operator training simulation for the process industries. In: atp international, vol. 1(1), pp. 24-36 (2003)

10. Urbas, L.: Entwicklung und Realisierung einer Trainings- und Ausbildungsumgebung zur Schulung der Prozeßdynamik und des Anlagenbetriebs im Internet. Fortschr. -Ber. VDIReihe 810 Nr. 614. VDI-Verlag, Düsseldorf (1999)

11. Klein, G.A.: A Recognition-Primed Decision (RPD) Model of Rapid Decision Making. In: Klein, G.A., Oresanu, J., Calderwood, R., Zsambok, C.E. (eds.) Decision Making in Action: Models and Methods, pp. 138-147. Ablex Publishing, Norwood (1992)

12. Salas, E., Wilson, K.A., Priest, H.A., Guthrie, J.W.: Design, Delivery, and Evaluation of training systems. In: Salvendy, G. (ed.) Handbook of human factors and ergonomics, 3rd edn., pp. 472-512. John Wiley \& Sons, New Jersy (2006)

13. Vogel-Heuser, B.: Automation in wood and paper industry. In: Nof, S.Y. (ed.) Handbook of Automation. Springer, New York (2008)

14. Pantförder, D., Vogel-Heuser, B.: Versuchsdesign für eine vergleichende Evaluation des Operator-Trainings bei der Visualisierung von Prozessdaten in einer 2D- und 3DUmgebung. In: Useware, Verfahrens- und Produktionstechnik, Engineering, Informationspräsentation und Interaktionskonzepte, VDI/VDE-Berichte 2041, pp. 143-154. VDIVerlag, Düsseldorf (2008)

15. Bortz, J.: Statistik für Sozialwissenschaftler. Springer, Berlin (1999) 